\title{
Immunopathogenesis of AIDS
}

\section{Alexandra M. Ortiz, BA, and Guido Silvestri, MD}

\author{
Corresponding author \\ Guido Silvestri, MD \\ Department of Pathology and Laboratory Medicine, University of \\ Pennsylvania School of Medicine, 705 Stellar-Chance Laboratories, \\ 422 Curie Boulevard, Philadelphia, PA 19104, USA. \\ E-mail: gsilvest@mail.med.upenn.edu \\ Current Infectious Disease Reports 2009, 11:239-245 \\ Current Medicine Group LLC ISSN 1523-3847 \\ Copyright (C 2009 by Current Medicine Group LLC
}

Twenty-five years after the discovery of HIV as the cause of AIDS, tremendous progress has been made in reducing the morbidity and mortality associated with this disease. However, there is still neither a cure nor a vaccine for HIV infection. This shortcoming is likely a consequence of our incomplete understanding of the mechanisms of AIDS pathogenesis, particularly with regard to the interaction between the virus and the host immune system. In this article we review a series of recent advances in specific areas of HIV immunopathogenesis research: virus-host cell interactions; immune responses to HIV; chronic immune activation; and natural simian immunodeficiency virus infections. Finally, we discuss the potential implications of these findings for HIV/AIDS therapy and vaccines.

\section{Introduction}

Primate lentiviral infections, including HIV infection of humans and simian immunodeficiency virus (SIV) of numerous nonhuman primate species, are characterized by a complex and highly dynamic interaction between the virus and the host immune system. This interaction ultimately leads to a severe state of immunodeficiency, AIDS, in humans and macaques (which are recent and experimental hosts for HIV and SIV infections), but does not result in disease in more than 40 African nonhuman primates naturally infected with SIV, such as sooty mangabeys, African green monkeys, and mandrills. The interaction between primate lentiviruses and host immune systems has at least four main aspects that profoundly influence AIDS immunopathogenesis and thus continue to be the focus of intense research: 1) these viruses replicate in immune cells (ie, activated $\mathrm{CD}^{+} \mathrm{T}$ cells and macrophages); 2) the immune response to these viruses appears to suppress virus replication somewhat (via antiviral neutralizing antibodies and CD8 ${ }^{+}$T-cell-mediated antiviral immune responses) while also paradoxically favoring virus replication by increasing the number of available target cells; 3 ) these viruses have a tremendous ability to escape host immune responses due to the peculiar molecular structure of their envelope proteins, tremendous genetic variability and flexibility, and various active immune evasion strategies; and 4) in the absence of effective immune control, the presence of chronic, generalized immune activation becomes a factor associated with (and likely leading to) progression to AIDS. Because of this complexity, the representation of HIV/AIDS pathogenesis has shifted from models that are mainly focused on the cytopathic role of the virus (ie, its direct killing of infected cells) to more comprehensive models that encompass the complexity of the virus-immune system interaction (immunopathogenesis) and incorporate chronic immune activation as a key factor for disease progression. In this review, we summarize the most recent advances in HIV/ AIDS immunology and immunopathogenesis.

\section{Virus-Host Cell Interaction}

A series of important discoveries made over the past few years clarified some previously poorly understood aspects of the molecular interactions between primate lentiviruses and host cells. Perhaps the two most important recent advances were the description of the cytoplasmic body molecule TRIM5a as a key post-entry, pre-integration, cellular restriction factor that acts through inhibition of virion uncoating, and the discovery of the cellular cytidine deaminase APOBEC3G [1,2]. Vif mediates the degradation of APOBEC3G, which is otherwise incorporated into progeny virions and leads to lethal hypermutation of the viral genome upon infection of the new target cell. More recently, the complexity of the interaction between HIV and cellular factors was further emphasized in a study describing the results of a functional genomic screening using a large library of short interfering RNA (siRNA). This work allowed the identification of a large set of cellular genes whose expression is required for effective virus replication [3]. Although using a somewhat artificial experimental system (ie, HeLa-derived cells that are not the natural host for HIV), these findings provide a previously unknown estimate for the number of individual virus-host cell factors interactions occurring during productive HIV infection.

In recent months, another major breakthrough in this field was the discovery that the cellular protein CD317/ tetherin/BST-2 prevents the release of virions from the surface of infected cells $[4 \bullet \bullet, 5 \bullet \bullet]$. In turn, the effect of 
CD317/tetherin is inhibited by the virus protein Vpu (for HIV-1) or by the intracytoplasmic tail of Env (for HIV-2 and certain SIVs). This antiviral effect of CD317 has profound and intriguing implications for viral pathogenesis and generation of immune responses to HIV. CD317 is a type 1 interferon (IFN)-induced surface protein that has a peculiar structure characterized by a transmembrane domain as well as a glycosylphosphatidylinositol anchor domain. This structure makes CD317 particularly well suited to tether (hence, "tetherin") budding virions to the surface of cells. It remains to be addressed whether, and if so, how, the CD317/tetherin antiviral activity is synergic with the function of the calcium-modulating cyclophilin ligand (CAML) protein, another recently identified Vpuinteracting host cell factor that restricts HIV-1 release using an unknown mechanism [6]. From an immunologic point of view, it will be interesting to determine if CD317 is involved in the trapping of virions by dendritic cells (DCs) and the resulting enhanced transmission of HIV/SIV to activated $\mathrm{CD}^{+}{ }^{+} \mathrm{T}$ cells. CD317/tetherin may also play an important role in priming antigen-presenting cells (including DCs) and thus influence the type of adaptive immune response to HIV. Interestingly, a strong direct antiviral role of another type 1 IFN-induced protein, TRIM-22, was described, further emphasizing the importance of type 1 IFN-mediated innate responses in inhibiting HIV replication [7]. In all, these exciting findings provide a continually more accurate view of the viral life cycle and its interaction with cellular proteins that will eventually translate into the identification of new targets for antiviral therapy.

\section{Immune Responses to HIV/SIV}

Pathogenic HIV/SIV infections are typically associated with strong, readily detectable, and persistent virusspecific adaptive immune responses involving B cells (through the production of virus-specific antibodies) and $\mathrm{T}$ cells. Unfortunately, these immune responses almost invariably fail to control virus replication and thus do not protect from disease progression. The reasons behind this failure are complex, and include 1) the ability of the virus to mutate and escape immune responses; 2) the structure of the envelope protein, which allows the virus to avoid antibody-mediated neutralization; 3) the ability of some HIV/SIV gene products, in particular Nef, to interfere with antigen presentation; 4) the presence of a pool of immunologically "silent," latently infected cells; and 5) the fact that virus-specific CD4+ T-helper cells are preferentially targeted by the virus. The enormous task that the immune system confronts in trying to prevent lentiviral infection and/or contain virus replication after infection was recently emphasized by the much-publicized negative result of the first large-scale, phase $2 b$, clinical trial of a T-cell-based candidate AIDS vaccine (human adenovirus type 5 expressing HIV gag/nef/pol一the STEP trial, also known as the Merck V520-023 study) [8]. Of interest, significant advances have defined new mechanisms underlying the failure of the immune system to control HIV infection. For instance, it was proposed that the increased expression of certain "inhibitory" surface markers (ie, programmed death 1, PD-1 and cytotoxic Tlymphocyte antigen 4, CTLA-4) on the surface of T cells during chronic HIV infection is a strong correlate of the loss of control of virus replication and disease progression [9-14]. HIV-specific T-cell function can be partially restored in vitro when these inhibitory signals are blocked, and thus it was proposed that in vivo PD-1 and/or CTLA4 blockade may reenergize the exhausted immune system and result in better control of virus replication. It remains to be determined, however, whether and to what extent the expression of PD-1 and CTLA- 4 causes, rather than reflects, HIV/SIV-associated T-cell dysfunction. As such, the possibility that blocking inhibitory signals on $\mathrm{CD} 4{ }^{+} \mathrm{T}$ cells could result in the increased availability of target cells for the virus must be considered [15-17]. Furthermore, a recent study in SIV-infected sooty mangabeys revealed that increased PD-1 expression is temporally associated with increased expression of immune activation markers during acute infection and that the resolution of this acute activation is accompanied by PD-1 down-regulation [18].

An area of research that is crucial for the design of an effective HIV/AIDS vaccine is the identification of immunologic correlates of protection from virus replication and disease progression. Studies of HIV-infected humans or SIV-infected macaques that spontaneously and persistently suppress virus replication below detectable limits (elite controllers, EC) may potentially provide important insights. However, it is still unclear to what extent the EC phenotype is caused by strong immune responses to HIV/SIV as opposed to low virus fitness and/or specific host genetic factors (ie, CCR5 genotype, CCL3L1 copy number). This complexity was emphasized by a recent report that failed to identify clear correlates of immune protection in a large group of EC SIV-infected rhesus macaques that carried the protective Mamu-B*08 and $\mathrm{B} * 17$ major histocompatibility complex (MHC) class 1 alleles [19]. Even more provocative is the suggestion that control of viremia itself is not sufficient to avoid $\mathrm{CD}^{+} \mathrm{T}$-cell depletion and progression to AIDS in a subset of ECs $[20 \bullet \bullet]$. In this context, one major but often ignored problem is that it is still largely unknown what proteins of HIV/SIV are targeted by cytotoxic T lymphocyte (CTL) responses early enough to elicit an effective block of virus replication at the level of the individual infected cell. Sacha et al. [21•] recently used an elegant in vitro experimental system to address this issue. In this study, these authors showed that HIV Gag protein may be the best target for CTLs because it can be recognized on infected cells in the context of MHC class 1 molecules before HIV integration and de novo viral protein expression. More recently, the same authors discovered that even within the same HIV antigen (ie, Gag) there is a priority of presentation among individual epitopes [22]. The importance of Gag as a target for protective CTL responses was 


\begin{tabular}{|c|c|}
\hline \multirow[t]{2}{*}{ Study } & Type of activation \\
\hline & Direct \\
\hline $\begin{array}{l}\text { Kiepiela et al. [23], Betts et al. [24], Manuel et al. [25], } \\
\text { Almeida et al. [26] }\end{array}$ & T-cell recognition of HIV epitopes_-adaptive immune response \\
\hline Funderburg et al. [34], Meier et al. [35] & $\begin{array}{l}\text { TLR stimulus in response to HIV infection-innate } \\
\text { immune response }\end{array}$ \\
\hline \multirow[t]{3}{*}{ Arthos et al. [33] } & Envelope-mediated signaling through $\alpha 4 \beta 7$-integrin \\
\hline & Loss of T-regulatory cells \\
\hline & Indirect \\
\hline Brenchley et al. [36], Ancuta et al. [37], Raffatellu et al. [38] & Microbial translocation across weakened mucosal barrier \\
\hline Mueller et al. [16] & Cytokine production (IL-2, IL-7, IL-15) \\
\hline Gottlieb et al. [29] & Opportunistic infections \\
\hline 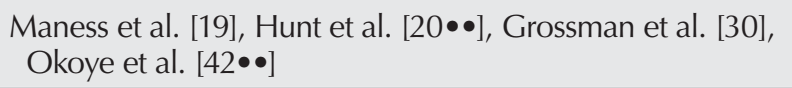 & Homeostatic proliferation in response to $\mathrm{CD}^{+}{ }^{+} \mathrm{T}$-cell depletion \\
\hline IL_interleukin; TLR-Toll-like receptor. & \\
\hline
\end{tabular}

also suggested by a study reporting a significant correlation between Gag-specific CTL responses and viral load (as well as an inverse correlation between Env-specific responses and viremia) in a large cohort of HIV-infected individuals from South Africa [23]. Clearly, the field of HIV immunology remains haunted by a basic chicken-and-egg issue: are the ostensibly better immune responses observed in association with low viral load causing this EC phenotype or, alternatively, does preservation of the immune system due to other factors make these HIV-specific T-cell responses appear to be more effective? It is, however, becoming apparent that a combination of multifunctionality, high avidity, increased clonal turnover, and Gag-focused responses may capture the key features of CTL responses associated with a more benign course of infection [24-26].

Given our incomplete understanding of the antiviral role of CTL responses during HIV/SIV infections, substantial research efforts have been devoted to the study of other cell types, such as natural killer (NK) cells, macrophages, and $\gamma-\delta$ T cells. Of interest, it was shown that certain combinations of NK cell receptors from the KIR3DL locus with HLA-B subtypes, particularly the activating KIR3DS1 receptor with its presumed ligand HLA-Bw4, have been associated with strong antiviral effects in vitro and slow progression to AIDS in epidemiologic studies $[27,28]$.

\section{Chronic Immune Activation}

The somewhat paradoxical observation that the clinically devastating immunodeficiency following HIV infection occurs in the context of major immune system activation was made in the very first description of AIDS [29]. Over the past few years, many observations led to the formulation of AIDS pathogenesis models in which HIVassociated chronic, generalized immune activation plays a central role in disrupting immune system homeostasis
[30-32]. More recently, significant advances were made in addressing two central questions in this area of research: 1) what causes HIV/SIV-associated immune activation? and 2) why is this immune activation so disruptive to immune homeostasis?

The mechanisms underlying HIV-associated immune activation are very complex and include 1) direct effects by the virus and its gene products (eg, Env, Tat); 2) innate and adaptive immune responses to viral antigens; 3) immune responses to secondary antigens, including opportunistic pathogens (eg, Epstein-Barr virus, cytomegalovirus, Candida albicans); 4) bystander immune activation induced by cytokines and other factors; 5 ) the failure of immunoregulatory circuits (ie, T-regulatory cells); and 6) a response to the translocation of microbes from the intestinal lumen into the systemic circulation [32] (Table 1). A potential role for HIV in directly inducing immune activation was proposed to result from the previously unrecognized ability of HIV Env to bind to the adhesion molecule $\alpha 4 \beta 7$-integrin [33]. Intriguingly, this effect of Env would not be limited to $\mathrm{CD}^{+}{ }^{+} \mathrm{T}$ cells but would also involve $\mathrm{CD} 8^{+} \mathrm{T}$ cells, which are known to be hyperactivated during HIV infection. The ability of HIV to activate large numbers of T cells in vivo has long puzzled scientists. Recent work conducted in the laboratories of Altfeld and Lederman may provide an explanation for this T-cell hyperactivation. These groups described how HIV can activate large numbers of $\mathrm{T}$ cells following stimulation of DCs via binding to various Tolllike receptors (TLR) [34,35]. In particular, it was shown that the single-stranded RNA of HIV-1 encodes multiple uridine-rich regions that act functionally as TLR-7/8 ligands on plasmacytoid dendritic cells [35]. Although the sequence of events involving innate and adaptive immune activation is still poorly defined, it is becoming clear that HIV and SIV infections may lead to stimulation of $\mathrm{T}$ cells (including those that are specific for other antigens) by unconventional mechanisms-distinct from the direct, T-cell receptor 


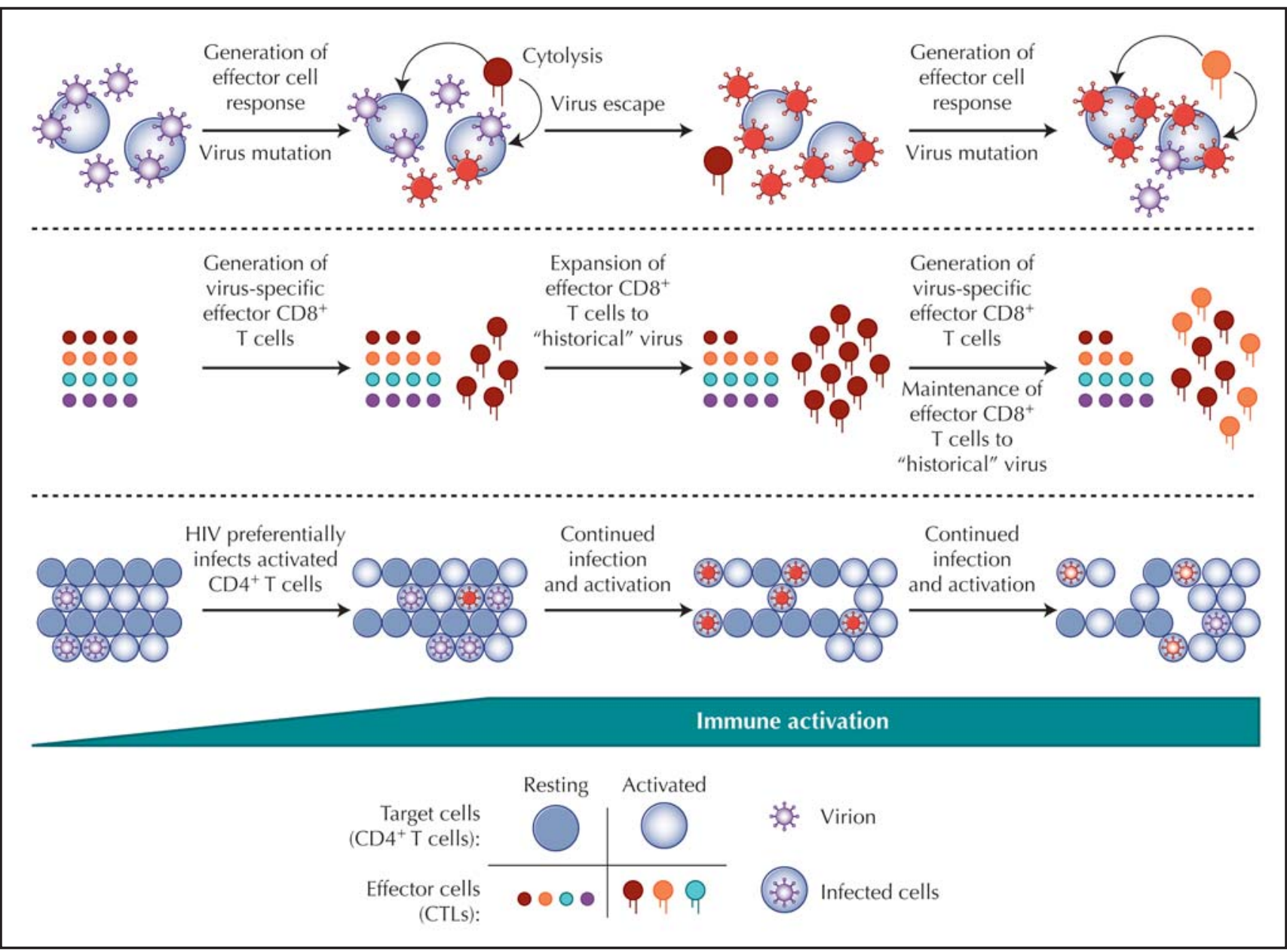

Figure 1. Chronic immune activation and the progression to immune exhaustion. HIV infection of activated CD4 ${ }^{+} \mathrm{T}$ cells leads to the expansion of $\mathrm{CD}^{+}$effector T cells (CTLs) specific for the originally acquired virus. CD4+ $\mathrm{T}$-cell depletion occurs as a result of virus-mediated cytopathicity, CTL-mediated cytolysis, and bystander immune activation and apoptosis. CD4 ${ }^{+} \mathrm{T}$ cells proliferate and become increasingly activated (as described in Table 1), heightening their susceptibility to viral infection. As mutations accrue within the host's virus populations, CTL responses lose the ability to effectively combat the majority of virions, and new responses must be generated, perpetuating the cycle of immune activation and ongoing $\mathrm{CD} 4^{+} \mathrm{T}$-cell depletion. Over many years, the ability to generate and expand new $\mathrm{CD} 4^{+}$and $\mathrm{CD} 8^{+} \mathrm{T}$ cells declines, accelerating the progression to AIDS.

(TCR)-mediated recognition of HIV/SIV antigens. Microbial translocation from the intestinal lumen to the systemic circulation is a well-recognized cause of immune activation and disease progression during pathogenic HIV/SIV infections [36]. The importance of this phenomenon was recently emphasized in an article suggesting a link between microbial translocation and neuropathogenesis via increased transport of HIV to the central nervous system by activated macrophages [37]. Subsequently, a series of studies conducted independently by various laboratories suggested a key role for the newly described subset of CD4+ T cells-Th17 cells-in the immunopathogenesis of AIDS [38-40]. Th17 cells are defined based on the production of cytokines such as IL-17 and IL-22 as well as their homing to mucosa-associated lymphoid tissues (MALT), where they mediate strong antimicrobial immunity [41]. During pathogenic HIV/SIV infections, CD4+ Th17 cells are preferentially depleted in the MALT (but not blood) of infected individuals, thus providing a potential new pathogenic mechanism for the HIV-associated mucosal immune dysfunction that leads to microbial translocation and chronic immune activation.

Significant progress has also been made in understanding how chronic immune activation negatively affects the preservation of the $\mathrm{CD}^{+}{ }^{+} \mathrm{T}$-cell pool to ultimately cause progression to AIDS (Fig. 1). In an elegant set of experiments performed in the laboratory of Louis Picker, it was shown that in chronically SIV-infected rhesus macaques, the key marker of disease progression is the depletion of central memory $\mathrm{CD}^{+}{ }^{+} \mathrm{T}$ cells $[42 \bullet \bullet]$. This observation showed for the first time that although effector memory $\mathrm{CD}^{+}{ }^{+} \mathrm{T}$ cells are the main target of the virus (and thus the cell population that is most immediately affected by SIV replication), progression to AIDS is largely determined by the destruction, failing production, and gradual decline of central memory $\mathrm{CD}^{+} \mathrm{T}$ cells. In another intriguing study, the preservation of the central memory T-cell pool was correlated, in a subset of EC HIV-infected individuals, with the inactivation of the 
FOXO3a intracellular signaling pathway [43•]. Collectively, these studies identify new biologic links between the abnormal activation of $\mathrm{CD}^{+} \mathrm{T}$ cells (particularly those of central memory cells) and the overall demise of the CD4+ $\mathrm{T}$-cell pool during HIV/SIV infection. The potential dangers of increasing immune activation while conducting interventions aimed at enhancing the immune responses to HIV/SIV have been predominantly emphasized by studies in which interleukin15 or an anti-CTLA-4 were administered to SIV-infected rhesus macaques [15-17]. In both instances, treatment may result in increasing virus replication (most likely due to increased availability of target cells) and consequent accelerated course of disease. An important implication of these studies is that they emphasize the potential pitfalls of interventions aimed at reversing in vivo the HIV-associated immune dysfunction by manipulating inhibitory receptors.

\section{Natural SIV Infection}

In stark contrast to the pathogenic HIV infection of humans and SIV infection of macaques, SIV infection in its natural hosts-African nonhuman primates, such as sooty mangabeys, African green monkeys, and mandrills-is typically nonpathogenic, with the hosts living an apparently normal lifespan after becoming infected [32]. It is increasingly recognized that understanding the mechanisms responsible for the AIDS resistance of natural SIV hosts will provide important insights regarding the pathogenesis of HIV infection and AIDS. Several early studies showed that natural SIV hosts remain asymptomatic and preserve healthy levels of $\mathrm{CD}^{+} \mathrm{T}$ cells despite persistently high levels of virus replication. Another peculiar feature of natural SIV infections is that they appear to be consistently associated with low levels of immune activation. Recent studies have significantly advanced this field by showing that natural SIV infection has an impact on CD4+ ${ }^{+}$-cell homeostasis and by elucidating some of the causes underlying the immune attenuated profile of natural SIV hosts.

Two articles reporting experiments conducted in sooty mangabeys and African green monkeys showed that acute SIV infection in these "nonprogressive" hosts is similar to pathogenic HIV/SIV infections in that it is also associated with rapid and severe depletion of mucosal $\mathrm{CD}^{+}{ }^{+} \mathrm{T}$ cells $[44,45]$. That natural SIV infections are not necessarily silent immunologically was further confirmed by the observation that profound systemic $\mathrm{CD} 4{ }^{+} \mathrm{T}$-cell depletion is observed in a subset of SIV-infected sooty mangabeys [46,47]. These CD4-low, SIV-infected sooty mangabeys do not show any signs of AIDS, suggesting that in this species, $\mathrm{CD} 4^{+} \mathrm{T}$ cells are more dispensable and/or that immune activation is a sine qua non for disease progression during primate lentiviral infections. Of additional interest, in sooty mangabeys and African green monkeys infected with SIV, the average in vivo lifespan of infected cells is similar to that observed in pathogenic HIV/SIV infections $[48,49]$. This finding strongly suggests that the relatively benign course of SIV infection in natural hosts is not due to lower intrinsic cytopathicity by the virus. Previous studies of SIV-specific T-cell responses in sooty mangabeys also indicated that the AIDS resistance of these animals is highly unlikely to be related to better immune control of virus replication [50]. Collectively, these reports emphasize that the apathogenicity of natural SIV infections cannot be explained simply by lower virus replication, reduced $\mathrm{CD} 4^{+}$ T-cell destruction, and/or better immune responses, but requires a further understanding of the specific dynamics of the host-virus interaction in these species.

Several current studies focus intently on the mechanisms by which natural SIV hosts consistently avoid chronic immune activation. These mechanisms are clearly complex and may vary widely across different monkey species. A few common themes have recently emerged, however, including the absence of microbial translocation [36], a decreased type 1 IFN response to TLR ligands by plasmacytoid DCs [51,52], the ability of SIV Nef to downmodulate CD3-TCR [53,54], and the presence of lower levels of CCR 5 on CD4+ $\mathrm{T}$ cells [55]. This last observation is of interest because it is consistent with a model wherein reduced CCR5 expression protects hosts from HIV disease progression independent of the role of CCR 5 as a coreceptor for virus entry $[56 \bullet, 57]$. Although we are still far from a complete understanding of why natural SIV infections are nonpathogenic, it is likely that further advances in this area of research will continue to provide useful clues to the mechanisms of AIDS pathogenesis in HIV-infected humans.

\section{Conclusions}

In this article we summarized a series of key advances made over the past few years in understanding HIV immunopathogenesis. Many of these findings are of particular interest because of their implications for HIV therapy and vaccines. The substantial improvement in understanding the molecular interaction between HIV/SIV and host cells may define new targets for antiviral therapy. Drugs that disrupt the virus-cell interactions required for HIV replication by acting on a particular cellular protein may represent an interesting new class of antiviral agents for which the mutability of the virus may not be a serious limiting factor. Our growing body of knowledge about the pathogenic role of chronic immune activation in determining HIV/SIV disease progression suggests that scientists in the field should more critically investigate the potential beneficial effects of interventions aimed at blunting chronic immune activation. These interventions may prove particularly useful in accelerating the recovery of immune function (by restoring and/or rebalancing the CD4+ ${ }^{+}$-cell pool), especially once virus replication has been successfully suppressed using conventional antiretroviral drugs. In terms of HIV/AIDS vaccine development, the key message emerging from the most recent studies is that much more needs to be learned about the interaction 
between HIV and the host immune system before we can reasonably expect to have a safe and effective AIDS vaccine. Although perhaps sobering, this message suggests that the optimal use of the available resources may require more emphasis on basic discovery and less emphasis on initiating clinical trials for candidate AIDS vaccines that have little to no chance of succeeding.

\section{Disclosure}

No potential conflicts of interest relevant to this article were reported.

\section{References and Recommended Reading}

Papers of particular interest, published recently, have been highlighted as:

- Of importance

$\bullet \quad$ Of major importance

1. Stremlau M, Owens CM, Perron MJ, et al.: The cytoplasmic body component TRIM5alpha restricts HIV-1 infection in Old World monkeys. Nature 2004, 427:848-853.

2. Harris RS, Liddament MT: Retroviral restriction by APOBEC proteins. Nat Rev Immunol 2004, 4:868-877.

3. Brass AL, Dykxhoorn DM, Benita Y, et al.: Identification of host proteins required for HIV infection through a functional genomic screen. Science 2008, 319:921-926.

4.• Neil SJ, Zang T, Bieniasz PD: Tetherin inhibits retrovirus release and is antagonized by HIV-1 Vpu. Nature 2008, 451:425-430.

This study identified the previously described BST-2 protein as the cellular protein responsible for tethering HIV virions to the surface of infected cells. The authors defined BST-2/tetherin's role in counteracting virion release and described a previously unrecognized role for the HIV protein Vpu in counteracting tetherin's activity and thus favoring the release of virions.

5.• Van Damme N, Goff D, Katsura C, et al.: The interferon-induced protein BST-2 restricts HIV-1 release and is downregulated from the cell surface by the viral Vpu protein. Cell Host Microbe 2008, 3:245-252.

This study identified the previously described BST-2 protein as the cellular protein responsible for tethering HIV virions to the surface of infected cells. The authors defined BST-2/tetherin's role in counteracting virion release and described a previously unrecognized role for the HIV protein Vpu in counteracting tetherin's activity and thus favoring the release of virions.

6. Varthakavi V, Heimann-Nichols E, Smith RM, Sun Y: Identification of calcium-modulating cyclophilin ligand as a human host restriction to HIV-1 release overcome by Vpu. Nat Med 2008, 14:641-647.

7. Barr S, Smiley J, Bushman F: The interferon response inhibits HIV particle production by induction of TRIM22. PLoS Pathog 2008, 4:e1000007.

8. Watkins DI, Burton DR, Kallas EG, Moore JP: Nonhuman primate models and the failure of the Merck HIV-1 vaccine in humans. Nat Med 2008, 14:617-621.

9. Sauce D, Almeida JR, Larsen M, et al.: PD-1 expression on human CD8 T cells depends on both state of differentiation and activation status. AIDS 2007, 21:2005-2013.

10. Zhang J, Zhang Z, Wang X, et al.: PD-1 up-regulation is correlated with HIV-specific memory CD8+ T-cell exhaustion in typical progressors but not in long-term nonprogressors. Blood 2007, 109:4671-4678.

11. Petrovas C, Casazza JP, Brenchley JM, et al.: PD-1 is a regulator of virus-specific CD8+ T cell survival in HIV infection. J Exp Med 2006, 203:2281-2292.
12. Trautmann L, Janbazian L, Chomont N, et al.: Upregulation of PD-1 expression on HIV-specific CD8+ T cells leads to reversible immune dysfunction. Nat Med 2006, 12:1189-1202.

13. Day CL, Kaufmann DE, Kiepiela P, et al.: PD-1 expression on HIV-specific T cells is associated with T-cell exhaustion and disease progression. Nature 2006, 443:350-354.

14. Kaufmann DE, Kavanagh DG, Pereyra F, et al.: Upregulation of CTLA-4 by HIV-specific CD4+ T cells correlates with disease progression and defines a reversible immune dysfunction. Nat Immunol 2007, 8:1246-1254.

15. Hryniewicz A, Boasso A, Edghill-Smith Y, et al.: CTLA-4 blockade decreases TGF-beta, IDO, and viral RNA expression in tissues of SIVmac251-infected macaques. Blood 2006, 108:3834-3842.

16. Mueller YM, Do DH, Altork SR, et al.: IL-15 treatment during acute simian immunodeficiency virus (SIV) infection increases viral set point and accelerates disease progression despite the induction of stronger SIV-specific CD8+ T cell responses. J Immunol 2008, 180:350-360.

17. Cecchinato V, Tryniszewska E, Ma ZM, et al.: Immune activation driven by CTLA- 4 blockade augments viral replication at mucosal sites in simian immunodeficiency virus infection. J Immunol 2008, 180:5439-5447.

18. Estes JD, Gordon SN, Zeng M, et al.: Early resolution of acute immune activation and induction of PD-1 in SIVinfected sooty mangabeys distinguishes nonpathogenic from pathogenic infection in rhesus macaques. J Immunol 2008, 180:6798-6807.

19. Maness NJ, Yant LJ, Chung C, et al.: Comprehensive immunological evaluation reveals surprisingly few differences between elite controller and progressor Mamu-B*17positive simian immunodeficiency virus-infected rhesus macaques. J Virol 2008, 82:5245-5254.

20.• Hunt PW, Brenchley JMB, Sinclair E, et al.: Relationship between T-cell activation and CD4(+) T cell count in HIV-seropositive individuals with undetectable plasma HIV RNA levels in the absence of therapy. J Infect Dis 2008, 197:126-133.

This article showed that even among ECs, immune activation remains higher than in uninfected individuals or HIV progressors treated with antiretroviral drugs. This immune activation was associated with increased CD $8^{+} \mathrm{T}$-cell proliferation and microbial translocation and was inversely correlated with CD4+ T-cell count irrespective of viral load.

21.• Sacha JB, Chung C, Rakasz EG, et al.: Gag-specific CD8+ $T$ lymphocytes recognize infected cells before AIDS-virus integration and viral protein expression. J Immunol 2007, 178:2746-2754.

Gag-specific CD8+ T-cell responses were associated with control of viral replication, although Gag is transcribed late in viral replication. The authors demonstrated that effective $\mathrm{CD} 8^{+} \mathrm{T}$-cell responses directed against multiple Gag epitopes can be generated within 2 hours of virus infection, before integration of the virus genome and Nef-mediated MHC-I down-regulation. This finding illustrated that $\mathrm{CD} 8^{+} \mathrm{T}$-cell responses can be generated before productive infection and highlighted the importance of inducing Gag-specific responses with prophylactic vaccines.

22. Sacha JB, Reynolds MR, Buechler MB, et al.: Differential antigen presentation kinetics of CD8+ T cell epitopes derived from the same viral protein. J Virol 2008, 82:9293-9298.

23. Kiepiela P, Ngumbela K, Thobakgale C, et al.: CD8+ T-cell responses to different HIV proteins have discordant associations with viral load. Nat Med 2007, 13:46-53.

24. Betts M, Nason M, West S, et al.: HIV nonprogressors preferentially maintain highly functional HIV-specific CD8+ T cells. Blood 2006, 107:4781-4789.

25. Manuel ER, Charini WA, Sen P, et al.: Contribution of T-cell receptor repertoire breadth to the dominance of epitope-specific CD8+ T-lymphocyte responses. J Virol 2006, 80:12032-12040. 
26. Almeida JR, Price DA, Papagno L, et al.: Superior control of HIV-1 replication by CD8+ T cells is reflected by their avidity, polyfunctionality, and clonal turnover. J Exp Med 2007, 204:2473-2485.

27. Martin MP, Qi Y, Gao X, et al.: Innate partnership of HLA-B and KIR3DL1 subtypes against HIV-1. Nat Genet 2007, 39:733-740.

28. Alter G, Martin MP, Teigen N, et al.: Differential natural killer cell-mediated inhibition of HIV-1 replication based on distinct KIR/HLA subtypes. J Exp Med 2007, 204:3027-3036.

29. Gottlieb MS, Schroff R, Schanker HM, et al.: Pneumocystis carinii pneumonia and mucosal candidiasis in previously healthy homosexual men: evidence of a new acquired cellular immunodeficiency. N Engl J Med 1981, 305:1425-1431.

30. Grossman Z, Meier-Schellersheim M, Paul WE, Picker LJP: Pathogenesis of HIV infection: what the virus spares is as important as what it destroys. Nat Med 2006, 12:289-295.

31. Sodora DL, Silvestri GS: Immune activation and AIDS pathogenesis. AIDS 2008, 22:439-446.

32. Silvestri GS, Paiardini M, Pandrea I, et al.: Understanding the benign nature of SIV infection in natural hosts. J Clin Invest 2007, 117:3148-3154.

33. Arthos J, Cicala C, Martinelli E, et al.: HIV-1 envelope protein binds to and signals through integrin alpha4beta7, the gut mucosal homing receptor for peripheral T cells. Nat Immunol 2008, 9:301-309.

34. Funderburg N, Luciano AA, Jiang W, et al.: Toll-like receptor ligands induce human $\mathrm{T}$ cell activation and death, a model for HIV pathogenesis. PLOS ONE 2008, 3:e1915.

35. Meier A, Alter G, Frahm N, et al.: MyD88-dependent immune activation mediated by human immunodeficiency virus type 1-encoded Toll-like receptor ligands. J Virol 2007, 81:8180-8191.

36. Brenchley JMB, Price DA, Schacker TW, et al.: Microbial translocation is a cause of systemic immune activation in chronic HIV infection. Nat Med 2006, 12:1365-1371.

37. Ancuta P, Kamat A, Kunstman K, et al.: Microbial translocation is associated with increased monocyte activation and dementia in AIDS patients. PLoS ONE 2008, 3:e2516.

38. Raffatellu M, Santos RL, Verhoeven DE, et al.: Simian immunodeficiency virus-induced mucosal interleukin-17 deficiency promotes Salmonella dissemination from the gut. Nat Med 2008, 14:421-428.

39. Cecchinato V, Trindade CJ, Laurence A, et al.: Altered balance between Th17 and Th1 cells at mucosal sites predicts AIDS progression in simian immunodeficiency virus-infected macaques. Mucosal Immunol 2008, 1:279-288.

40. Brenchley J, Paiardini M, Knox K, et al.: Differential Th17 CD4 T-cell depletion in pathogenic and nonpathogenic lentiviral infections. Blood 2008, 112:2826-2835.

41. Dong C: TH17 cells in development: an updated view of their molecular identity and genetic programming. Nat Rev Immunol 2008, 8:337-348.

42.• Okoye A, Meier-Schellersheim M, Brenchley JMB, et al.: Progressive CD4+ central memory T-cell decline results in CD4+ effector memory insufficiency and overt disease in chronic SIV infection. J Exp Med 2007, 204:2171-2185.

Using the rhesus macaque model of SIV infection, the authors illustrated that chronic SIV infection resulted in profound and continuous depletion of effector memory $\mathrm{CD}^{+} \mathrm{T}$ cells at effector sites (mucosal tissues). This study demonstrated that $\mathrm{CD} 4^{+}$depletion is ongoing and that replenishment of effector memory CD4+ $\mathrm{T}$ cells at effector sites depends on their ongoing generation from central memory $\mathrm{CD}^{+} \mathrm{T}$ cells. The inability of effector $\mathrm{CD} 4^{+}$memory cells to repopulate mucosal tissues occurred subsequent to loss of the central memory $\mathrm{CD}^{+} \mathrm{T}$-cell population, suggesting that although total CD4+ T-cell depletion is necessary for progression to AIDS, disease progression may be determined largely by the rate of central memory $\mathrm{CD}^{+}{ }^{+} \mathrm{T}$-cell loss.
43. Van Grevenynghe J, Procopio FA, He Z, et al.: Transcription factor FOXO3 controls the persistence of memory CD4(+) T cells during HIV infection. Nat Med 2008, 14:266-274.

These authors previously demonstrated that phosphorylation of FOXO3a (pFOXO3a) is responsible for conferring cytokine-mediated survival to central memory $\mathrm{CD} 4{ }^{+} \mathrm{T}$ cells, thus contributing to this cell population's prolonged survival and resistance to apoptosis. In this study, the authors showed that HIV-infected ECs have increased levels of pFOXO3a in central and effector memory $\mathrm{CD}^{+}{ }^{+} \mathrm{T}$ cells. Increased pFOXO3a was associated with long-term persistence of these memory populations; furthermore, abrogation of the FOXO3a pathway in memory cells of HIV-infected progressors extended the survival of these cells in vitro.

44. Gordon SN, Klatt NR, Bosinger SE, et al.: Severe depletion of mucosal CD4+ T cells in AIDS-free simian immunodeficiency virus-infected sooty mangabeys. J Immunol 2007, 179:3026-3034.

45. Pandrea IV, Gautam R, Ribeiro RM, et al.: Acute loss of intestinal CD4+ $T$ cells is not predictive of simian immunodeficiency virus virulence. J Immunol 2007, 179:3035-3046.

46. Milush JM, Stefano-Cole K, Schmidt K, et al.: Mucosal innate immune response associated with a timely humoral immune response and slower disease progression after oral transmission of simian immunodeficiency virus to rhesus macaques. J Virol 2007, 8:6175-6186.

47. Sumpter B, Dunham RM, Gordon SN, et al.: Correlates of preserved CD4(+) T-cell homeostasis during natural, nonpathogenic simian immunodeficiency virus infection of sooty mangabeys: implications for AIDS pathogenesis. J Immunol 2007, 178:1680-1691.

48. Gordon SN, Dunham RM, Engram JC, et al.: Short-lived infected cells support virus replication in sooty mangabeys naturally infected with simian immunodeficiency virus: implications for AIDS pathogenesis. J Virol 2008, 82:3725-3735.

49. Pandrea IVP, Ribeiro RM, Gautam R, et al.: Simian immunodeficiency virus SIVagm dynamics in African green monkeys. J Virol 2008, 8:3713-3724.

50. Dunham RM, Pagliardini P, Gordon S, et al.: The AIDS resistance of naturally SIV-infected sooty mangabeys is independent of cellular immunity to the virus. Blood 2006, 108:209-217.

51. Diop OM, Ploquin MJ, Mortara L, et al.: Plasmacytoid dendritic cell dynamics and alpha interferon production during simian immunodeficiency virus infection with a nonpathogenic outcome. J Virol 2008, 82:5145-5152.

52. Mandl JN, Barry AP, Vanderford TH, et al.: Divergent TLR7 and TLR9 signaling and type I interferon production distinguish pathogenic and nonpathogenic AIDS virus infections. Nat Med 2008, 14:1077-1087.

53. Schindler M, Münch J, Kutsch O, et al.: Nef-mediated suppression of $\mathrm{T}$ cell activation was lost in a lentiviral lineage that gave rise to HIV-1. Cell 2006, 125:1055-1067.

54. Schindler M, Schmökel J, Specht A, et al.: Inefficient Nefmediated downmodulation of $\mathrm{CD} 3$ and MHC-I correlates with loss of CD4+T cells in natural SIV infection. PLOS Pathog 2008, 4:e1000107.

55. Pandrea IVP, Apetrei C, Gordon SN, et al.: Paucity of CD4+CCR5+ T cells is a typical feature of natural SIV hosts. Blood 2007, 109:1069-1076.

56. Ahuja SK, Kulkarni H, Catano G, et al.: CCL3L1-CCR5 genotype influences durability of immune recovery during antiretroviral therapy of HIV-1-infected individuals. Nat Med 2008, 14:413-420.

The aim of this study was to determine the effect of particular genetic factors in HIV-infected individuals on immune reconstitution in response to antiretroviral therapy. The authors found that individuals with a high CCL3L1 (MIP1 1 ) gene copy number and a previously defined, nondetrimental CCR 5 phenotype were associated with improved immune reconstitution following therapy, independent of the role these genetic factors play during acute infection.

57. Dolan MJ, Kulkarni H, Camargo JF, et al.: CCL3L1 and CCR5 influence cell-mediated immunity and affect HIVAIDS pathogenesis via viral entry-independent mechanisms. Nat Immunol 2007, 8:1324-1336. 